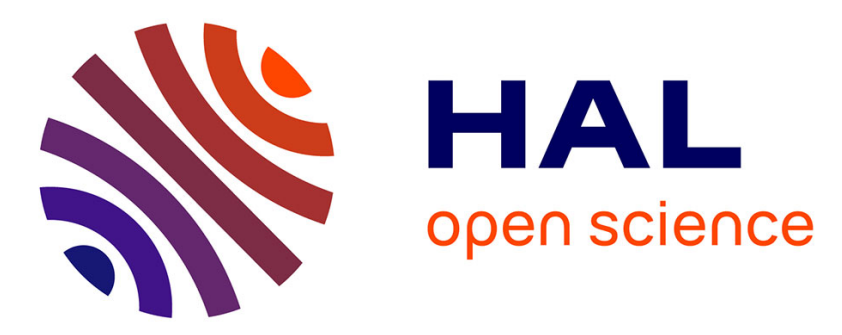

\title{
Evolution des taux d'azote et de carbone organiques dans un sol nu après 15 ans d'enfouissement de paille sous différentes conditions
}

\author{
Robert Morel, Catherine Chabouis, Serge Bourgeois, Thérèse Lasnier
}

\section{- To cite this version:}

Robert Morel, Catherine Chabouis, Serge Bourgeois, Thérèse Lasnier. Evolution des taux d'azote et de carbone organiques dans un sol nu après 15 ans d'enfouissement de paille sous différentes conditions. Agronomie, 1981, 1 (1), pp.7-17. hal-00884217

\section{HAL Id: hal-00884217 https://hal.science/hal-00884217}

Submitted on 1 Jan 1981

HAL is a multi-disciplinary open access archive for the deposit and dissemination of scientific research documents, whether they are published or not. The documents may come from teaching and research institutions in France or abroad, or from public or private research centers.
L'archive ouverte pluridisciplinaire HAL, est destinée au dépôt et à la diffusion de documents scientifiques de niveau recherche, publiés ou non, émanant des établissements d'enseignement et de recherche français ou étrangers, des laboratoires publics ou privés. 


\section{Evolution des taux d'azote et de carbone organi- ques dans un sol nu après 15 ans d'enfouissement de paille sous différentes conditions}

Robert MOREL, Catherine CHABOUIS, Serge BOURGEOIS

avec la collaboration technique de Thérèse LASNIER

Chaire de Physico-Chimie et Science du Sol, Institut National Agronomique Paris-Grignon, 78850 ThivervalGrignon.

\section{RÉSUMÉ}

Azote organique du sol, Carbone organique du sol, Enfouissement de paille, Paille préhumifiée, Sol nu,

Evolution à long terme.
Ce mémoire relate les premiers résultats d'une expérience parcellaire mise en place en 1959 en sol maintenu sans culture pour étudier les effets d'enfouissement de pailles de céréales et d'azote minéral sous différentes conditions. Les traitements appliqués au sol sont les suivants :

1. - sans azote minéral, sans paille

2. - sans azote minéral, avec paille

3. - sans azote minéral, avec paille préhumifiée

4. - avec azote minéral, sans paille

5. - avec azote minéral, avec paille

6. - avec paille préhumifiée en présence d'azote minéral.

Les apports de pailles (blé) et d'azote minéral sont respectivement par ha et par an de $7000 \mathrm{et} 70 \mathrm{~kg}$.

La préhumification, réalisée dans des cuves de ciment isothermes, apporte des modifications importantes :

- perte de 60 p. 100 environ du carbone de la paille (avec ou sans azote);

- gain d'azote dans le cas de préhumification sans apport d'azote $(2,4 \mathrm{~g}$ en moyenne d'azote par $\mathrm{kg}$ de paille);

- perte importante d'azote (40 p. 100 environ) dans le cas de la paille préhumifiée en présence d'azote minéral.

Les observations sur le sol sont les suivantes:

- dans tous les cas, les teneurs en azote et en carbone organiques du sol se sont abaissées entre 1959 et 1977. La paille, préhumifiée ou non, limite les pertes (fig. 3 et 4 ); la préhumification ne modifie pas l'effet de la paille de façon significative.

Les apports d'azote diminuent également les pertes d'azote et de carbone organiques du sol mais de façon beaucoup moins marquée que la paille:

- l'étude statistique des résultats montre que les effets de la fumure azotée et de l'enfouissement de pailles sont rigoureusement additifs (absence d'interaction significative);

- les rendements de l'azote et du carbone organiques apportés par la paille en azote et carbone organiques du sol ont été évalués : ils sont très différents pour chacun des deux éléments (tabl. 7). Ainsi, dans le traitement $\mathrm{n}^{\circ} 2$ (sans azote, avec paille), le rendement du carbone de la paille est de 6 p. 100 environ, celui de l'azote de 57 p. 100. L'écart est plus marqué encore avec l'apport d'azote. La préhumification abaisse le rendement de l'azote, mais ne modifie pas de façon sensible celui du carbone de la paille mise en fermentation.

\section{ABSTRACT}

Soil organic nitrogen, Soil organic carbone, Straw manuring Prehumificated straw, Bare soil,

Long term evolution.
Evolution of the organic nitrogen and carbon rates in an uncovered soil after 15 years of straw manuring under different conditions.

This report gives the first results of a plot experiment set up in 1959 on an untilled soil to study the effects of manuring with cereal straw and mineral nitrogen under different conditions. The treatments applied to the soil are the following ones:

1. - without mineral nitrogen and without straw

2. - without mineral nitrogen, with straw

3. - without mineral nitrogen with prehumified straw

4. - with mineral nitrogen, without straw

5. - with mineral nitrogen, with straw

6. - with straw prehumified in presence of mineral nitrogen.

The straw (wheat) and mineral nitrogen supplies reach respectively 7 tons and $70 \mathrm{~kg}$ per ha and year. The prehumification carried out in isothermal cement tanks brings important modifications :

- loss of about 60 p. 100 of the straw carbon (with or without nitrogen) ;

- gain of nitrogen in the case of prehumification without nitrogen supply $(2.4 \mathrm{~g}$ nitrogen per $\mathrm{kg}$ of straw in average) ; 
- important nitrogen loss (about $40 \mathrm{p} .100$ ) in the case of straw prehumified in presence of mineral nitrogen. The observations concerning the soil are the following ones:

- in all cases the soil contents in organic nitrogen and carbon have decreased between 1959 and 1977 . The straw, prehumified or not, limits the losses (fig. 3 and 4). Prehumification does not modify the straw effect significantly. Nitrogen supplies reduce also the losses of the soil organic nitrogen and carbon but much less than straw does;

- the statistical analysis of the results reveals that the effects of nitrogen supply and straw manuring are strictly additive :

- the yields in soil organic nitrogen and carbon obtained with the organic nitrogen and carbon provided by the straw have been estimated : they greatly differ for each of the two elements (tabl. 7). In treatment 2 for instance (without nitrogen and straw) the yield of the straw carbon reaches about 6 p. 100, that of nitrogen 57 p. 100. The divergence is still more considerable with nitrogen supply. The prehumification lowers the yield of nitrogen but does not modify the yield of the carbon in the straw which is submitted to fermentation.

\section{INTRODUCTION}

L'évolution d'une substance organique introduite dans le sol se déroule d'une façon plus ou moins rapide selon le composant considéré et son état de liaison avec la fraction minérale.

Au plan quantitatif et envisagée dans son ensemble, cette évolution a donné lieu à de nombreuses recherches. Certaines, conduites sur des quantités limitées de sol, permettent de suivre, pendant des temps généralement assez courts, de l'ordre de quelques mois à quelques années par exemple, l'ampleur de la destruction d'un substrat organique introduit dans un sol donné (FÜHR et SAUERBECK, 1968 ; SAUERBECK et GonZalEZ, 1976). D'autres travaux, engagés sur des durées plus longues, envisagent l'étude de l'évolution des teneurs du sol en matière organique dans des conditions normales de culture ; ils concernent en particulier l'effet de l'apport de résidus de récolte de diverses natures, parfois plus ou moins transformés avant leur incorporation au sol (Demolon et BuRGEvin, 1940, 1941 ; Simon, 1960 ; Delas, 1971 ; I.T.C.F., 1976 ; DUTIL, MULlER et BALLIF, 1980 ; JUSTE, 1980 ; MULLER et REMMY, 1980).

Dans son ensemble et sous un climat tempéré, l'évolution de la matière organique dans le sol se traduit par des variations quantitatives très lentes, requérant pour leur mise en évidence de façon sûre des expérimentations d'une durée suffisamment longue. Au plan agronomique, cette lenteur d'évolution se traduit par une incertitude sur les conséquences, à long terme, d'une modification du rythme des restitutions organiques au sol. La question s'est notamment posée, naguère, pour de nombreuses exploitations de l'Ile de France devenues essentiellement céréalières, et dans lesquelles l'enfouissement traditionnel de fumier d'étable avait été supprimé depuis plusieurs décennies; le retour au sol des seuls résidus de récoltes, enfouis en totalité ou en partie, appelait alors la question de savoir si cet apport organique se révélait suffisant pour que la production végétale ne fut pas diminuée.

Il apparaissait dès lors opportun d'implanter un essai parcellaire dans le but d'étudier l'influence des résidus de récoltes (paille de blé) apportés au sol sous différentes conditions. Un dispositif était ainsi mis en place en 1959.

L'expérience, conduite en sol nu, affrait dans ces conditions l'avantage de ne pas introduire de systèmes racinaires peu accessibles à une détermination quantitative précise ; par ailleurs, des difficultés techniques de culture étaient évitées, consécutives à l'incorporation au sol de masses assez importantes de paille.

L'inconvénient de ce dispositif est que le sol peut présenter un comportement difficilement comparable à celui de sols cultivés. Toutefois, il s'est vite révélé que cet essai, dit des « 36 parcelles », par les résultats qu'il apporte, offrait à la réflexion des informations du plus grand intérêt sur l'évolution générale de la matière organique dans les sols.

\section{MATÉRIEL ET MÉTHODES}

\section{Le dispositif parcellaire. Différents traitements}

Le dispositif, situé sur le domaine du Centre de Grignon, au fond du vallon du rû de Gally, est implanté sur un sol brun colluvial faiblement lessivé ; le substratum calcaire est à un mètre environ de la surface ; l'ensemble du profil est normalement drainé. La couche arable présente la granulométrie moyenne suivante :

$\begin{array}{ll}\text { Argile } & \text { (particules }<0,002 \mathrm{~mm} \text { ) } 290 \text { p. } 1000 . \\ \text { Limon } & \text { (particules comprises entre } 0,002 \text { et } \\ \text { Sable fin } & \text { (p2,02 mm) 250 p. } 1000 . \\ & \text { (particules comprises entre } 0,02 \text { et } 0,2 \\ \text { Sable grossier } 420 \text { p. } 1000 . \\ \text { (particules comprises entre } 0,2 \text { et } 2 \\ \text { mm) } 40 \text { p. } 1000 .\end{array}$

Le taux de calcaire varie entre 3 et 10 p. 100 , le $\mathrm{pH}$ (eau) s'établit entre 8 et 8,5 .

La teneur en carbone du sol se situait au début de l'essai entre 13 et 15 p. 1000 , la teneur en azote entre 1,4 et 1,5 p. 1000 .

L'environnement climatique du dispositif (période 1957-1974) se caractérise par les données ci-après :

- pluviométrie annuelle : $642 \mathrm{~mm}$

- évapotranspiration potentielle : $650 \mathrm{~mm}$

- bilan hydrique excédent hivernal (octobre-mars) : $179 \mathrm{~mm}$ déficit estival : $235 \mathrm{~mm}$

- températures moyennes mensuelles (sous abri) minimum : $-3{ }^{\circ} \mathrm{C}$ (janvier 1963) maximum : $+25,5^{\circ} \mathrm{C}$ (juillet 1959).

L'essai place en comparaison des parcelles avec ou sans apport d'azote minéral, avec ou sans incorporation de paille soumise ou non à une préhumification préalable. Les facteurs étudiés, en complément du seul enfouissement de paille, sont donc l'influence d'un apport d'azote minéral d'une part, la fermentation de la paille en l'absence ou en présence d'azote d'autre part.

Les six traitements suivants répondent aux préoccupations exprimées :

1. - parcelles sans azote, sans paille 
2. - parcelles sans azote, avec paille

3. - parcelles sans azote, avec paille préhumifiée

4. - parcelles avec azote, sans paille

5. - parcelles avec azote, avec paille

6. - parcelles avec paille préhumifiée en présence d'azote.

Le dispositif expérimental comprend 6 blocs de 6 parcelles carrées de $10 \mathrm{~m}^{2}$ chacune, séparées par des allées. Les 6 traitements sont répartis au hasard dans chacun des blocs.

Les quantités de paille et d'azote apportées annuellement par parcelle sont les suivantes:

$$
\begin{array}{ll}
\text { paille de blé : } & 7 \mathrm{~kg}\left(70 \mathrm{q} / \mathrm{ha}^{-1}\right) \\
\text { azote : } & 0,070 \mathrm{~kg}\left(70 \mathrm{~kg} / \mathrm{ha}^{-1}\right) .
\end{array}
$$

L'azote est incorporé sous forme de nitrate d'ammonium.

Deux labours annuels sont effectués à la bêche (profondeur $25 \mathrm{~cm}$ ), l'un à la fín de l'automne (novembre), l'autre fin février. Le premier permet l'incorporation de la paille. préhumifiée ou non, coupée au préalable en fragments de 10 à $15 \mathrm{~cm}$ de longueur ; le second labour se révèle indispensable pour obtenir une homogénéisation satisfaisante de la paille dans le sol, difficile à réaliser en une seule opération. L'engrais azoté est incorporé au sol au moment du bêchage de fin d'hiver. Enfin, des binages répartis au cours de l'année assurent la propreté des parcelles.

\section{La préhumification de la paille}

La fermentation de la paille est conduite dans deux cuves cubiques $(0,90 \times 0,90 \times 0,90 \mathrm{~m})$, en ciment lissé, à double paroi et dont l'espace vide compris entre ces dernières est rempli de mâchefer. Le fond de chaque cuve, légèrement incliné, permet par un tuyau de plomb de recueillir le "purin" dans un récipient placé dans une fosse bétonnée. Les cuves sont, pendant la fermentation de la paille, fermées en permanence par un couvercle amovible non étanche. Cette réalisation simple, qui permet d'éviter de trop grandes déperditions de chaleur lors de la préhumification, a jusqu'ici donné toute satisfaction.

La fermentation de la paille se poursuit d'avril à novembre, l'une des cuves assurant la préhumification sans azote, l'autre avec apport d'azote. Dans ce dernier cas, l'azote est incorporé sous forme de nitrate d'ammonium dissous dans l'eau d'arrosage de la paille. Chaque cuve reçoit environ $50 \mathrm{~kg}$ de paille coupée en fragments de 10 à $15 \mathrm{~cm}$ de longueur puis tassée le plus possible ; des arrosages à l'eau déminéralisée sont effectués, chaque jour au début de l'opération puis plus espacés ; le purin est toujours recyclé, de l'eau étant ajoutée ou non selon le cas (les quantités de purin recueillies sont variables). La paille en cours de fermentation est homogénéisée 2 fois entre avril et novembre sur une aire bétonnée plane aménagée au pied des cuves. Un apport bactérien ne s'est pas révélé nécessaire.

La fabrication du "fumier artificiel » étant terminée, chaque parcelle du dispositif reçoit en fumier l'équivalent de $7 \mathrm{~kg}$ de paille apportés lors de la mise en fermentation.

\section{Echantillonnage du sol, méthodes analytiques}

L'échantillonnage du sol a été réalisé tous les trois ans depuis 1959 , le premier par mélange de 3 sondages, les suivants par mélange de 16 sondages pour chaque parcelle (profondeur $25 \mathrm{~cm}$ ).

Les taux d'azote total du sol, de la paille fermentée ou non, sont déterminés par la méthode de KJELDAHL. Le taux de carbone du sol est évalué selon la technique d'oxydation bichromique en milieu sulfurique de ANNE. La teneur en carbone des pailles et fumier est obtenue par oxydation dans l'oxygène et dosage du $\mathrm{CO}_{2}$ dégagé.

Les dosages d'azote et de carbone ont été testés sur deux parcelles pour lesquelles chaque sondage a fait l'objet d'une analyse individuelle. Le tableau suivant résume les résultats

\begin{tabular}{|c|c|c|c|c|c|c|}
\hline $\begin{array}{l}\text { Eléments } \\
\text { considérés }\end{array}$ & $\begin{array}{l}\text { Numéro de } \\
\text { la parcelle }\end{array}$ & $\begin{array}{l}\text { Nombre } \\
\text { de sondages } \\
\text { par parcelle }\end{array}$ & $\begin{array}{l}\text { Teneurs } \\
\text { moyennes } \\
\text { p. } 1000 \text { de } \\
\text { terre sèche }\end{array}$ & $\begin{array}{l}\text { Variance due } \\
\text { à la technique }\end{array}$ & $\begin{array}{l}\text { Variance due } \\
\text { à l'échantillonnage } \\
\text { intraparcellaire }\end{array}$ & $\begin{array}{c}\text { Intervalle de confiance } \\
(\mathrm{P}=0,01) \text { sur la valeur } \\
\text { moyenne parcellaire }\end{array}$ \\
\hline Azote...$\ldots \ldots \ldots$ & 111 & 16 & 1,11 & $3,29 \cdot 10^{-5}(1)$ & $123,9 \cdot 10^{-5}$ & $1,08-1,15$ \\
\hline Carbone ........... & 161 & 15 & 12,0 & $2,88 \cdot 10^{-2}(2)$ & $3,75 \cdot 10^{-2}$ & $11,7-12,2$ \\
\hline
\end{tabular}
obtenus :

Nombre de déterminations analytiques par échantillon (1): 2, (2): 4 .

\section{RÉSULTATS EXPÉRIMENTAUX}

Ils concernent d'une part les modifications observées sur la paille en fermentation, d'autre part l'évolution quantitative de l'azote et du carbone organiques du sol.

\section{Evolution de la paille au cours de la préhumification}

Cette évolution se traduit par un changement progressif de la coloration qui devient peu à peu brun noirâtre. Le volume apparent diminue considérablement, la densité du "fumier » en fin de fermentation étant légèrement supérieure dans le cas d'apport d'azote (exemple $1977: 0,62$ et $0,57)$.

Les températures des masses de paille mises en fermentation s'élèvent après quelques jours à des valeurs de l'ordre de 60 à $65^{\circ} \mathrm{C}$, sans que des écarts très sensibles soient observés entre les 2 cuves (avec ou sans apport d'azote). Elles s'abaissent ensuite assez rapidement, s'élevant à nouveau après la première homogénéisation (fig. 1).

L'évolution de la composition élémentaire de la paille au cours de la fermentation révèle, quant à elle, des modifications particulièrement nettes :

- perte de l'ordre de 60 p. 100 du carbone introduit par la paille, dans les deux types de traitements (avec ou sans azote) ;

- gain d'azote dans le cas de la préhumification sans apport d'azote, la quantité totale d'azote contenue dans le "fumier » atteignant 1,5 fois environ la quantité contenue dans la paille ; ce gain d'azote est de l'ordre de $2,4 \mathrm{~g}$ d'azote par $\mathrm{kg}$ de paille mise en fermentation;

- perte d'azote pour la paille préhumifiée en présence 


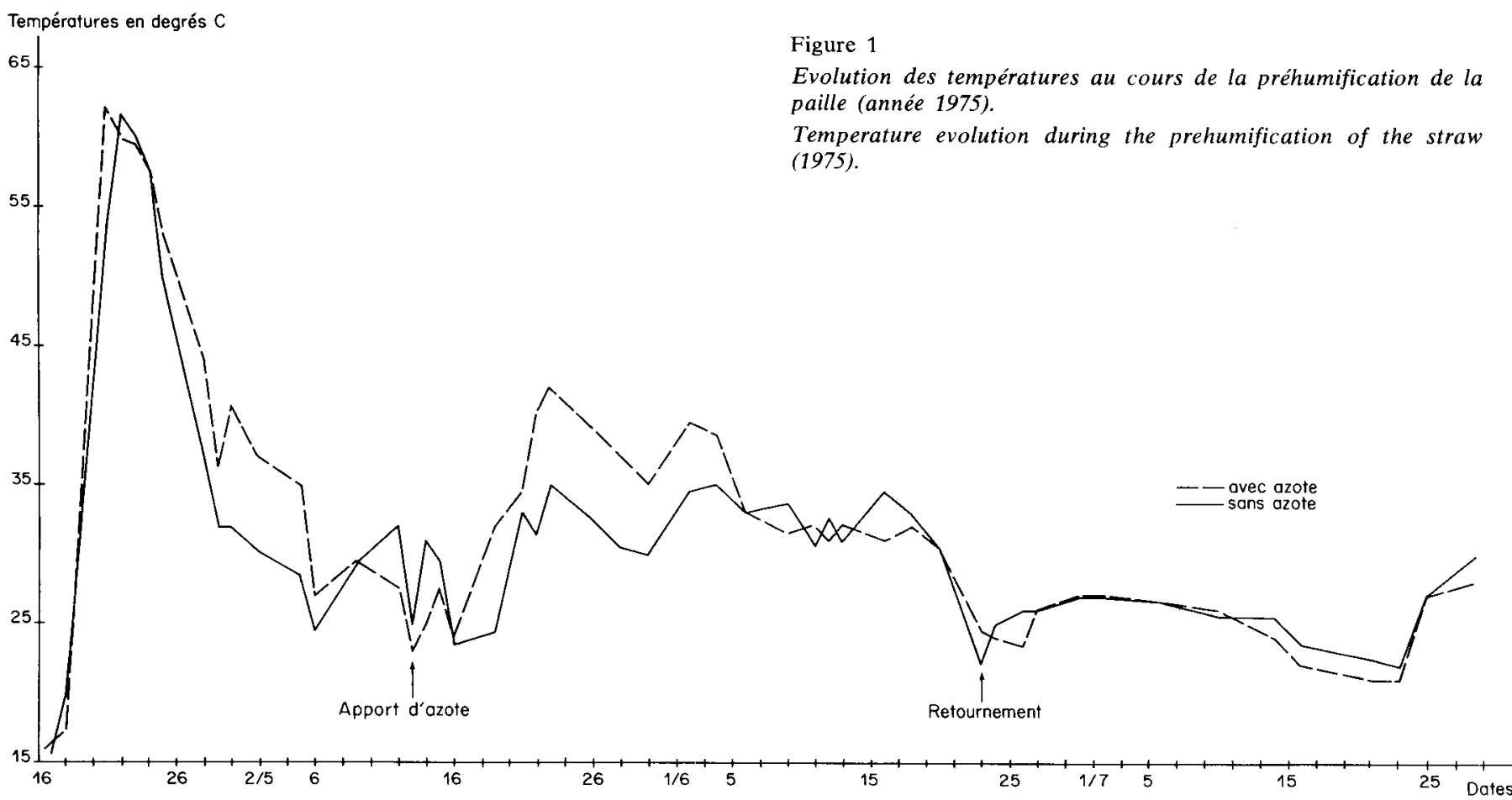

d'azote; la quantité de cet élément retrouvée dans le "fumier" atteint à peine 60 p. 100 de la quantité totale d'azote apportée (azote de la paille + azote minéral ajouté).

Le tableau 1 reproduit les résultats moyens obtenus qui, selon les années, peuvent varier dans des proportions notables selon les conditions de fabrication de la paille préhumifiée : les teneurs en azote des pailles mises en fermentation, notamment, se sont échelonnées entre 3,54 et 7,02 p. 1000 de paille séchée à $105^{\circ} \mathrm{C}$ (années 1977 et 1979). Néanmoins, les conclusions ci-dessus exprimées se retrouvent très régulièrement chaque année, ne laissant planer aucun doute sur leur validité.

Il résulte de ces observations que les pailles préhumifiées avec ou sans azote apportent en définitive au sol des quantités d'azote organique assez peu différentes : l'apport par les premières est en moyenne 1,15 fois plus élevé que celui des secondes.

\section{Evolution des teneurs en azote organique du sol}

L'évolution générale des teneurs en azote est représentée sur la figure 2. Pour la période comprise entre 1959 et 1962 , seuls deux traitements accusent une nette diminution (sans azote, sans paille et avec azote, avec paille) tandis que le taux d'azote ne varie pas ou varie peu pour les autres traitements.

L'interprétation des résultats de cette première période se révèle difficile, rendue plus délicate encore par le fait que ces résultats ont été acquis en 1959 sur un échantillonnage de 3 sondages, en 1962 sur un échantillonnage de 16 sondages.

Par contre, après 1962, l'évolution est caractérisée par une décroissance générale des teneurs en azote, quels que soient les traitements; toutefois, cette diminution n'apparaît pas régulière, sans qu'il soit possible de déceler les

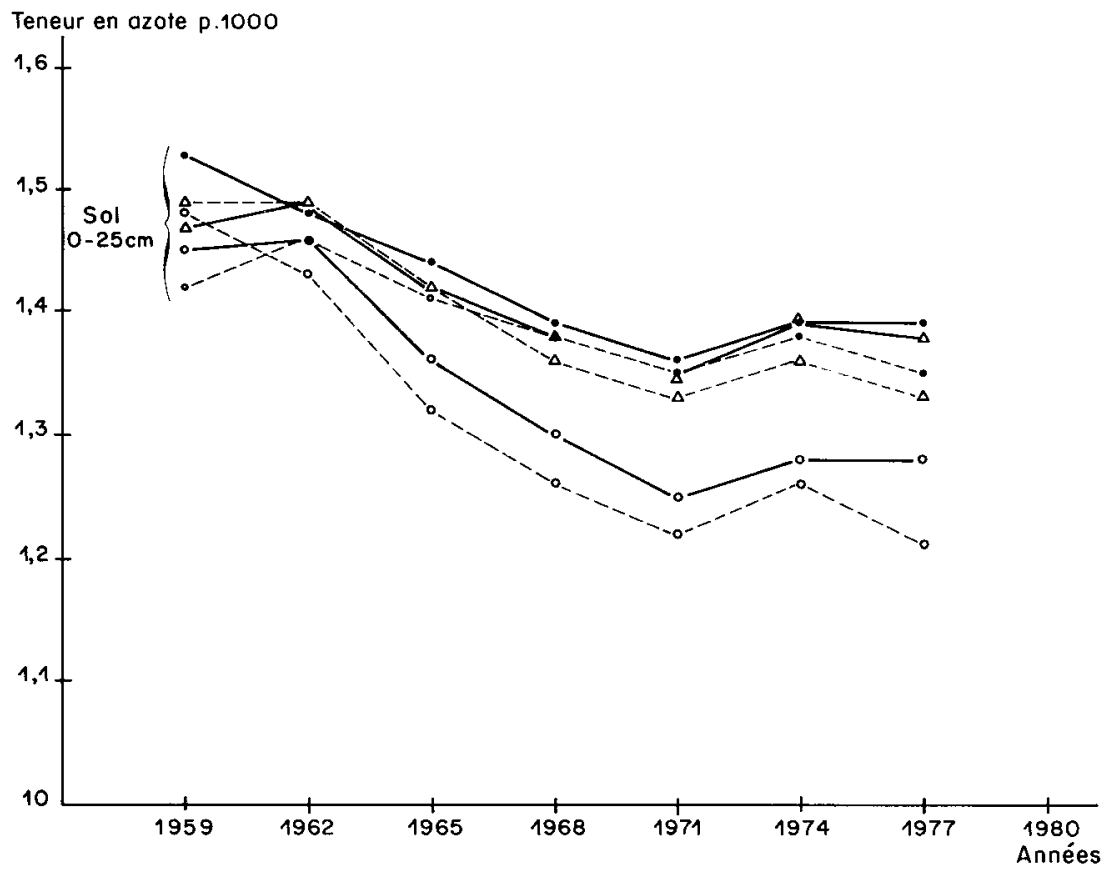

Figure 2

Evolution des taux d'azote organique du sol entre 1959 et 1977.

Evolution of the soil organic nitrogen amounts from 1959 to 1977.

sans azote (without nitrogen)

O-----O sans paille (without straw)

---- avec paille (with straw)

$\triangle---\triangle$ avec paille préhumifiée (with prehumificated straw)

avec azote (with nitrogen)

$\bigcirc-0$ sans paille (without straw)

$\longrightarrow$ avec paille (with straw)

$\triangle$ avec paille préhumifiée (with prehumificated straw). 
TABLEAU 1

Données et résultats relatifs à la fermentation de la paille (moyenne de 6 années)

Data and results concerning the fermentation of the straw

\begin{tabular}{|c|c|c|}
\hline & $\begin{array}{l}\text { Fermentation } \\
\text { sans azote }\end{array}$ & $\begin{array}{l}\text { Fermentation } \\
\text { avec azote }\end{array}$ \\
\hline Paille mise en fermentation $(\mathrm{kg})$.. & 46,08 & 46,3 \\
\hline $\begin{array}{r}\text { Teneur de la paille en azote } \\
(\mathrm{p} .1000)\end{array}$ & 5,19 & 5,19 \\
\hline Poids du fumier frais $(\mathrm{kg}) \ldots \ldots \ldots \ldots$ & 149,5 & 139,7 \\
\hline 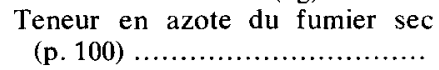 & 18,0 & 22,58 \\
\hline Poids de l'azote dans le fumier (g) & 349,7 & 405,5 \\
\hline $\begin{array}{l}\text { Azote total apporté (paille }+ \text { azote } \\
\text { minéral })(\mathrm{g})\end{array}$ & 239,6 & 703,2 \\
\hline Bilan (gain ou perte d'azote) (g) ... & $+110,1$ & $-297,7$ \\
\hline Bilan par $\mathrm{kg}$ de paille apporté $(\mathrm{g})$ & $+\quad 2,39$ & $-6,43$ \\
\hline Rapport $\frac{\text { azote dans le fumier }}{\text { azote total apporté }} \ldots .$. & 1,46 & 0,58 \\
\hline $\begin{array}{l}\text { Taux de carbone dans la paille } \\
\text { mise en fermentation ................... }\end{array}$ & 40,95 & 40,95 \\
\hline Carbone dans la paille $(\mathrm{kg}) \ldots \ldots \ldots$ & 18,87 & 18,96 \\
\hline Carbone dans le fumier $(\mathrm{kg}) \ldots \ldots$. & 7,57 & 6,86 \\
\hline Perte de carbone $(\mathrm{kg})$.................... & 11,30 & 12,10 \\
\hline Taux de perte en carbone .......... & 59,9 & 63,8 \\
\hline
\end{tabular}

causes de ce comportement. Le tableau 2 précise cette évolution. Le tableau 3 indique les pertes d'azote organique du sol et leur signification ainsi que le bilan de l'azote entre 1962 et 1977.

Une étude statistique des résultats a été réalisée d'une part sous forme d'une analyse de variance multivariable portant sur les 6 séries de mesures effectuées tous les ans de 1962 à 1977, par une analyse de variance année par année d'autre part.

Le résultat essentiel de cette étude est que les actions de la fumure minérale azotée et de l'enfouissement de pailles sont rigoureusement additives (absence d'interaction significative). Il est donc possible de considérer de façon indépendante les effets de l'apport d'azote et de l'incorporation de paille.

\subsection{Influence des apports de paille (tabl. 4)}

L'incorporation de paille au sol limite les pertes d'azote. Cette influence, exprimée sans ambiguitté sur la figure 3 , se manifeste de façon significative dès 1962 ; à partir de 1968 , les écarts entre les teneurs en azote demeurent sensiblement constants; la préhumification ne modifie pas l'effet de la paille.

L'influence de l'incorporation de pailles sur les pertes d'azote organique du sol, considérées entre 1962 et 1977 , est rapportée sur le tableau 3 . Les apports de paille et de paille préhumifiée diminuent respectivement les pertes de 284 et $180 \mathrm{~kg}$ en 15 ans (49 et 31 p. 100); mais remarquons que la différence des pertes entre les deux traitements n'est pas significative.

\subsection{Influence des apports d'azote (tabl. 4)}

Cette influence est beaucoup moins marquée sur les teneurs en azote organique du sol que dans le cas des pailles. Le test global est significatif, mais les effets n'apparaissent que pour certaines années, plus particulièrement en 1977.

Entre 1962 et 1977, l'apport d'azote a diminué les pertes d'azote organique du sol de $127 \mathrm{~kg}$ en 15 ans (26 p. 100) (tabl. 3).

\subsection{Bilan de l'azote (tabl. 3)}

Excepté pour le traitement sans azote et sans paille, les pertes totales d'azote subies par le sol entre 1962 et 1977 reflètent l'importance des apports du fait que ceux-ci sont supérieurs aux pertes propres du sol dans des proportions parfois importantes. La perte observée pour le traitement $\mathrm{n}^{\circ} 6$ (paille préhumifiée avec azote) paraît nettement inférieure à celle des traitements 4 et 5 . Mais il faut noter que lors de la préhumification, $634 \mathrm{~kg}$ d'azote ont disparu et n'ont donc pas été incorporés au sol ; sil'on tient compte de cette quantité, la perte totale pour le traitement $n^{\circ} 6$ s'établit à $2023 \mathrm{~kg}$.

\section{Evolution des teneurs en carbone organique du sol}

La figure 3 représente cette évolution. Contrairement aux variations observées sur les taux d'azote, à une exception

TABLEAU 2

Teneurs du sol en azote et en carbone organiques (p. 1000 de sol sec)

Soil organic nitrogen and organic carbon amounts (p. 1000 of dry soil)

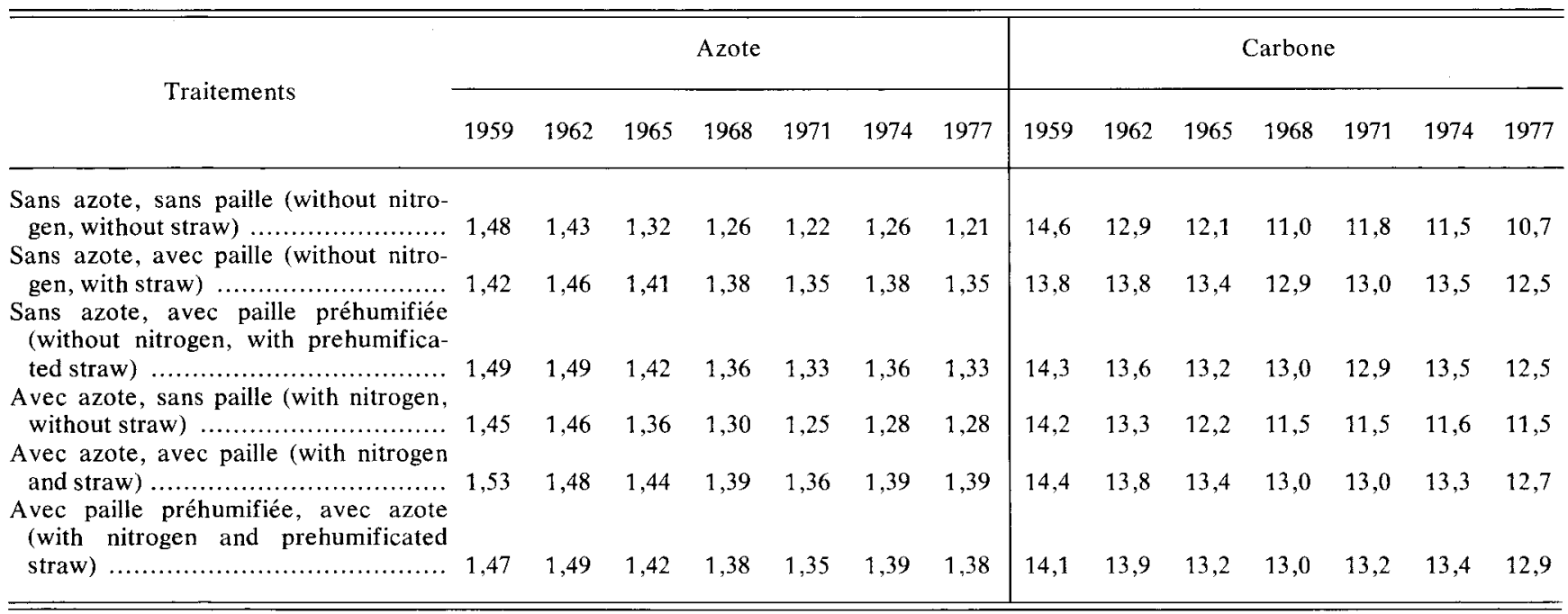




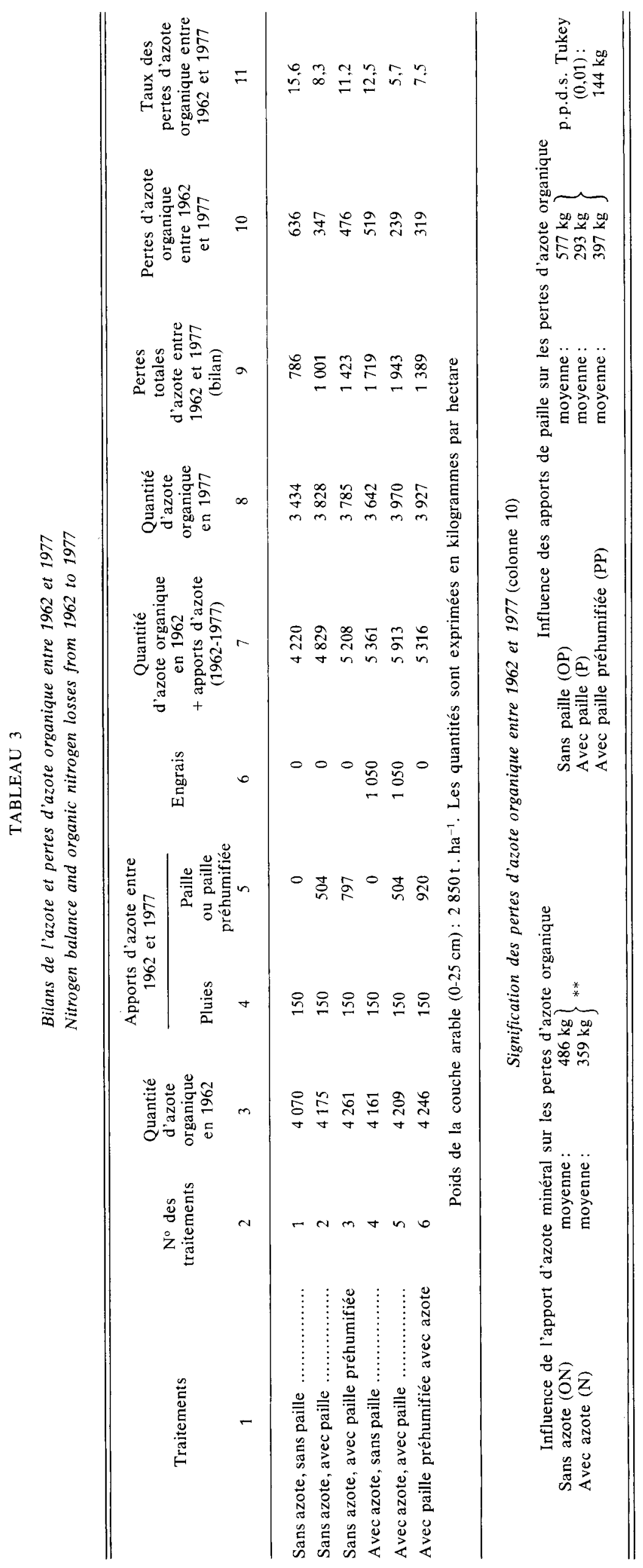




\section{TABLEAU 4}

Evolution des teneurs moyennes en azote organique du sol entre 1962 et 1977 (azote p. 1000 de terre sèche)

Evolution of soil organic nitrogen average amounts from 1962 to 1977 (p. 1000 of dry soil)

\begin{tabular}{|c|c|c|c|c|c|c|}
\hline \multirow{2}{*}{ Traitements } & \multicolumn{6}{|c|}{ Années } \\
\hline & 1962 & 1965 & 1968 & 1971 & 1974 & 1977 \\
\hline 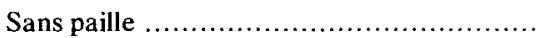 & 1,44 & 1,34 & 1,28 & 1,23 & 1,27 & 1,24 \\
\hline Avec paille $\ldots \ldots \ldots \ldots \ldots \ldots$ & 1,47 & 1,42 & 1,39 & 1,35 & 1,38 & 1,37 \\
\hline Avec paille préhumifiée ....... & 1,49 & 1,42 & 1,37 & 1,34 & 1,37 & 1,35 \\
\hline 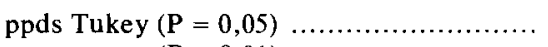 & 0,03 & 0,03 & 0,03 & 0,03 & 0,03 & 0,04 \\
\hline$(P=0,01) \ldots \ldots \ldots \ldots \ldots \ldots \ldots \ldots$ & - & 0,04 & 0,04 & 0,04 & 0,05 & 0,05 \\
\hline 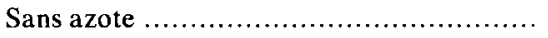 & 1,46 & 1,38 & 1,33 & 1,30 & 1,33 & 1,29 \\
\hline 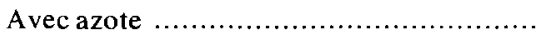 & 1,47 & 1,41 & 1,36 & 1,32 & 1,35 & 1,35 \\
\hline Signification de la différence $\ldots \ldots \ldots \ldots \ldots \ldots$ & - & $*$ & $*$ & $(*)$ & - & $* *$ \\
\hline
\end{tabular}

Signification du test $\mathrm{F}:{ }^{*} \mathrm{P}=0,01,{ }^{*} \mathrm{P}=0,05,\left({ }^{*}\right) \mathrm{P}=0,10$.

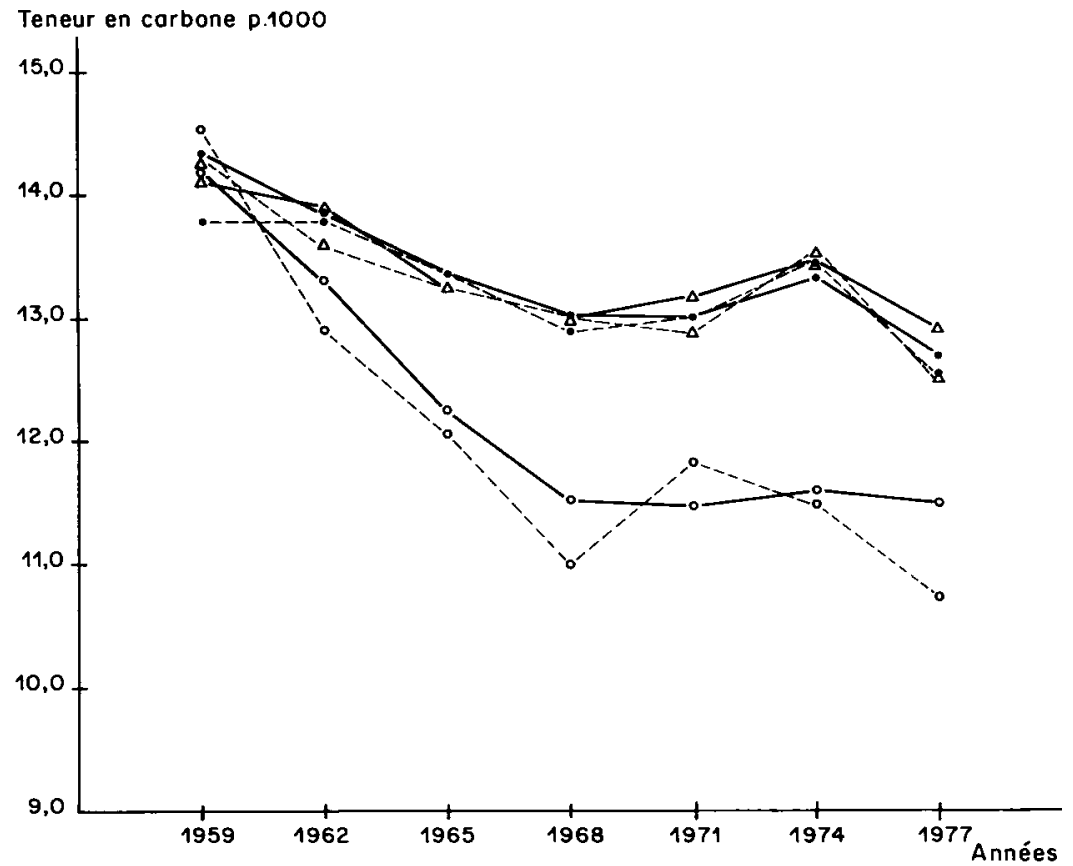

Figure 3

Evolution des taux de carbone organique du sol entre 1959 et 1977.

Evolution of the soil organic carbon amounts from 1959 to 1977 (see the notice fig. 2).

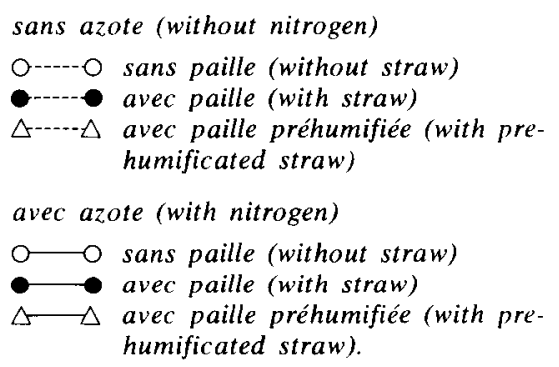

près (sans azote, avec paille), les teneurs en carbone organique s'abaissent dès 1959 , de façon particulièrement marquée en l'absence d'apport de paille. Cette diminution se poursuit après 1962, avec les irrégularités déjà remarquées pour l'azote et actuellement non interprétées.

Les tableaux 2 et 5 caractérisent cette évolution entre 1962 et 1977.

L'étude statistique des teneurs en carbone, conduite de la même façon que précédemment, permet de donner des résultats très comparables aux résultats obtenus pour l'azote :

- additivité des effets de la fumure minérale azotée et de l'enfouissement des pailles;

- diminution des taux de carbone limitée de façon hautement significative par les apports de paille, sans distinction significative entre la paille et la paille préhumi- fiée (tabl. 6). Cette influence de la paille se lit également sans ambiguité sur la figure 3. Considérés entre 1962 et 1977 , les apports de paille et de paille préhumifiée diminuent respectivement les pertes de 2223 et $2708 \mathrm{~kg}$ (39 et 48 p. 100). Mais notons que la différence des pertes entre les deux traitements n'est pas significative (tabl. 5);

- influence positive des apports d'azote sur la diminution des pertes, influence là encore beaucoup moins marquée que dans le cas de la paille mais globalement significative par le fait des écarts relevés en 1962 et 1977 (tabl. 6). Toutefois, si l'on considère comme référence l'année 1962, la perte de carbone estimée en 1977 n'est pas significativement diminuée par l'apport d'azote (tabl. 5), fait apparemment contradictoire aux résultats précédents. Cela est dû à ce que l'effet de l'azote s'était déjà manifesté dès 1962, mais ne s'est que peu accentué entre 1962 et 1977. 

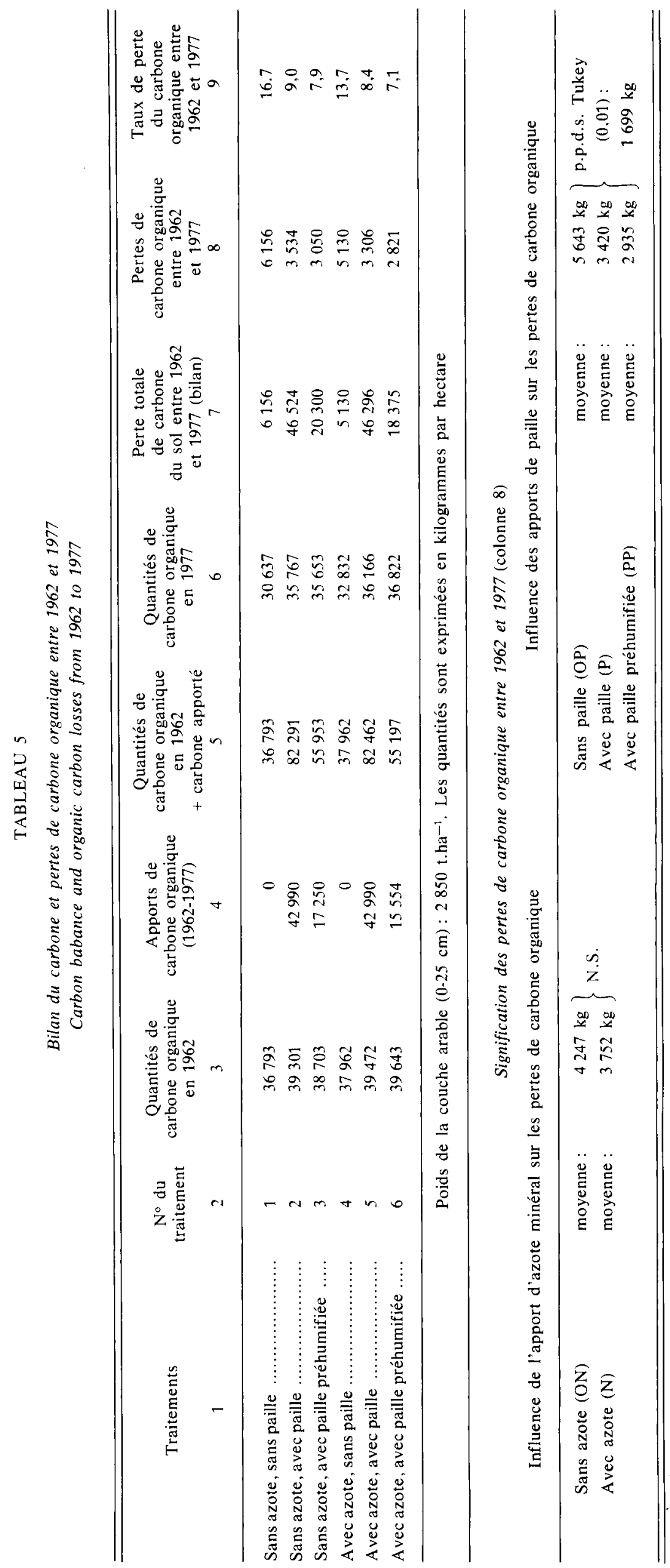
TABLEAU 6

Evolution des teneurs moyennes en carbone organique du sol entre 1962 et 1977 (carbone p. 1000 de terre sèche)

Evolution of soil organic carbon average amounts from 1962 to 1977 (p. 1000 of dry soil)

\begin{tabular}{|c|c|c|c|c|c|c|}
\hline \multirow{2}{*}{ Traitements } & \multicolumn{6}{|c|}{ Années } \\
\hline & 1962 & 1965 & 1968 & 1971 & 1974 & 1977 \\
\hline Sans paille. & 13,1 & 12,2 & 11,3 & 11,6 & 11,5 & 11,1 \\
\hline Avec paille & 13,8 & 13,4 & 13,0 & 13,0 & 13,4 & 12,6 \\
\hline Avec paille préhumifiée ......................... & 13,7 & 13,2 & 13,0 & 13,0 & 13,5 & 12,7 \\
\hline 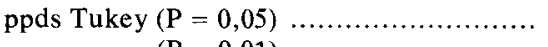 & 0,3 & 0,3 & 0,4 & 0,6 & 0,4 & 0,4 \\
\hline 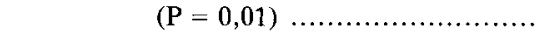 & 0,5 & 0,4 & 0,5 & 0,8 & 0,5 & 0,5 \\
\hline 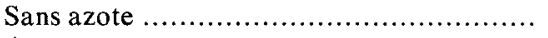 & 13,4 & 12,9 & 12,3 & 12,6 & 12,8 & 11,9 \\
\hline 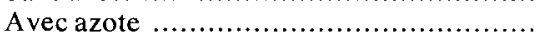 & 13,7 & 12,9 & 12,5 & 12,6 & 12,8 & 12,4 \\
\hline Signification de la différence $\ldots \ldots \ldots \ldots \ldots \ldots$ & * & - & - & - & - & $* *$ \\
\hline
\end{tabular}

De même que dans le cas de l'azote et pour les traitements comportant des apports organiques, le bilan du carbone (pertes totales entre 1962 et 1977) reflète très largement l'influence des apports qui sont quantitativement très supérieurs aux pertes propres du sol.

\section{Observations sur l'évolution de l'azote et du carbone organiques du sol}

Les courbes représentatives de l'évolution de l'azote et du carbone organiques se classent en deux groupes distincts selon que des pailles sont ou non incorporées au sol (fig. 2 et 3).

Pour chacun des deux groupes, une première partie des courbes traduit une baisse très notable des teneurs en azote et en carbone ; la seconde partie paraît indiquer le début d'une certaine stabilité ("palier»). Celle-ci, dans le cas des traitements avec apports organiques, permettrait de définir les niveaux azotés et carbonés du sol en équilibre dans les conditions de l'expérience.

Par contre, le « palier » correspondant aux traitements ne comportant pas d'apport organique apparaît moins explicable du fait qu'aucune masse carbonée n'est incorporée au sol: les courbes concernées devraient en effet dans ce cas tendre de plus en plus lentement vers une ordonnée sensiblement nulle.

Une suggestion peut être proposée quant à la relative stabilité observée après quelques années seulement: la première partie de la courbe se rapporterait à un épuisement assez rapide de la fraction labile de la matière organique du sol ; la partie suivante correspondrait, quant à elle, à la diminution très lente de la fraction organique stable, diminution qui ne pourra être mise en évidence de façon sûre qu'après un grand nombre d'années. Cette explication appelle un corollaire : si, après quelques années, il devenait possible de mieux préciser l'ordonnée du "palier», la courbe concernant le traitement sans apport d'azote et de paille devrait permettre, d'une façon approximative, de déterminer l'importance relative de la fraction organique labile du sol au début de l'expérience.

\section{Rendements de l'azote et du carbone organiques apportés au sol}

Les rendements en azote et carbone organiques du sol de l'azote et du carbone incorporés sous forme de paille préhumifiée ou non peuvent être estimés pour les divers traitements par référence aux parcelles témoins ne recevant aucun apport organique :

témoin 1 : traitement sans azote et sans paille témoin 4 : traitement avec azote et sans paille.

Pour les traitements 2 et 3 , sans apport d'azote, l'expression du rendement se rapporte uniquement à l'effet des pailles (témoin 1). Pour le traitement 5 , la référence au témoin 4 indique les rendements propres de l'azote et du carbone des pailles tandis que la référence au traitement 1 inclut dans le rendement l'effet de l'azote minéral apporté au sol (action additive). Le traitement 6 (avec paille préhumifiée avec azote) quant à lui, ne peut avoir comme référence le témoin 4, l'azote de ce témoin étant incorporé directement au sol.

Les résultats ainsi calculés (tabl. 7) suggèrent les conclusions suivantes :

- Les rendements sont différents pour l'azote et le carbone, les premiers étant généralement très supérieurs aux seconds : ainsi, le rendement de l'azote de la paille dans le traitement 2 est de 57 p. 100 , celui du carbone de 6 p. 100 ; cette différence s'accentue dans le traitement $n^{\circ} 5$ et plus sévèrement encore si l'on ne considère pour le carbone que l'effet propre de la paille $(4,2$ p. 100$)$.

- La préhumification diminue beaucoup le rendement de l'azote et augmente celui du carbone. Toutefois, si l'on choisit comme référence le carbone initialement contenu dans la paille mise en fermentation, les rendements du carbone sont alors sensiblement les mêmes pour la paille préhumifiée (6 à 7 p. 100).

- L'apport d'azote minéral ne modifie pas le rendement propre de l'azote de la paille (57 et 55 p. 100), mais diminue celui du carbone $(6,1$ et 4,2 p. 100$)$.

Cet ensemble d'observations sur les rendements du carbone et de l'azote organiques souligne de la façon la plus nette qu'il n'est pas indifférent, dans toute étude quantitative sur l'évolution des substances organiques apportées au sol, de choisir comme référence le carbone ou l'azote: chacun des deux éléments doit être, dans certaines démarches, considéré séparément.

\section{CONCLUSION}

L'expérimentation mise en place en 1959 sur un sol maintenu sans culture pour examiner l'effet sur le sol 


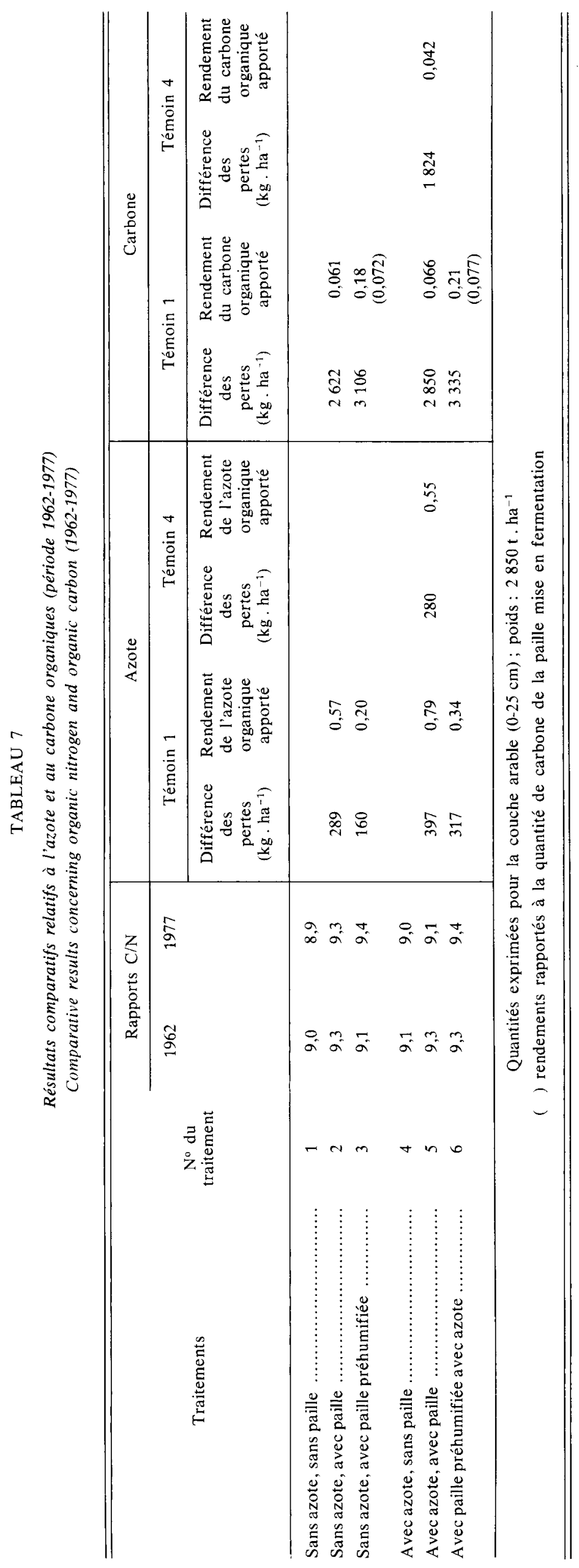


d'enfouissement de paille sous différentes conditions, permet de répondre à certaines questions sur la dynamique de l'azote et du carbone dans le sol.

Un apport important de paille, préhumifiée ou non, avec ou sans azote, n'assure pas le maintien des taux d'azote et de carbone du sol ; cette observation apparaît contradictoire au fait qu'avec des apports organiques moindres, des sols cultivés de même type et sous le même climat conservent ou même augmentent leurs teneurs en ces éléments. Cette comparaison incite à envisager et à souligner le rôle majeur des racines dans l'établissement du niveau organique du sol.

Les résultats acquis précisent un fait important: l'additivité des effets de l'azote et de l'enfouissement de paille dans la limitation des pertes de carbone et d'azote organiques du sol ; l'action de la paille est en cela très largement supérieure à celle de l'azote. Cette observation peut, entre autres, constituer l'un des éléments de décision dans l'établissement de plans expérimentaux relatifs à l'action sur le sol d'apports organiques et d'engrais azotés.
Par ailleurs, les différences très importantes observées sur les rendements de l'azote et du carbone organiques apportés doivent conduire à traiter séparément les deux éléments dans l'étude de certains problèmes relatifs à la dynamique de la matière organique dans le sol.

Reçu le 25 septembre 1980.

Accepté le 10 octobre 1980.

\section{REMERCIEMENTS}

Nous exprimons nos vifs remerciements à MM. C. JUSTE et J. C. Remy, respectivement des Stations d'Agronomie de Bordeaux et de Laon (I.N.R.A.), avec lesquels nous avons eu de fructueux échanges de vues et qui nous ont fait part de leurs observations sur ce travail, Madame P. Renaud, MM. Ph. Huet et J. F. Fourbet, respectivement des Chaires de Mathématiques et d'Agronomie de l'I.N.A. Paris-Grignon, qui nous ont largement apporté leur concours dans les interprétations statistiques exposées dans ce mémoire.

\section{REFERENCES BIBLIOGRAPHIQUES}

Delas J., 1971. Evolution des propriétés d'un sol sableux sous l'influence d'apports massifs et répétés de matières organiques de différentes origines. Ann. Agron., 22 (5), 585-610.

Demolon A., Burgevin H., 1940. Sur l'enfouissement direct des pailles de céréales dans le sol et ses répercussions. Ann. Agron., nouvelle série, $t$. $X, 357-365$.

Demolon A., Burgevin H., 1941. Humification des pailles et fabrication du fumier artificiel. Public. du Secrétariat d'Etat à l'Agriculture, Collection de monographies et mises au point publiées par les Stations et Laboratoire de Recherches Agronomiques, $2^{e}$ édition, 6-33.

Dutil P., Muller J., Ballif J. L., 1980. Pailles et énergie. Données actuelles sur le problème de l'enlèvement des pailles en Champagne crayeuse. Travaux de la Station de Science du Sol de Châlons-surMarne. Publication $n^{\circ} 76$.

Führ F., Sauerbeck D., 1968. Decomposition of wheat straw in the field on influenced by cropping and rotation. Isotopes and Radiation in Soil Organic-Matter Studies, Proceedings of a symposium. Vienne $1968,241-250$.
Juste M.C., 1980. Exportation maximale de biomasse et conservation de la fertilité des sols en zone tempérée: cas de l'enlèvement des pailles de céréales à des fins énergétiques. Publ. CENECA, Agriculture et Energie.

Muller J. C., Rémy J. C., 1980. Influence de l’azote minéral sur la cinétique de la décomposition des pailles. Commission des Commu nautés Européennes - Projet E : Energie à partir de la biomasse. Etude $\mathrm{n}^{\circ} 1$ : Protection des sols en régime d'exportation maximale de matière organique. Publ. I.N.R.A., janvier 1980, 6-21.

Sauerbeck D. R., Gonzalez, 1976. Field decomposition of carbon14-labelled plant residues in various soils of the federal Republic of Germany and Costa Rica. Soil Organic Matter Studies, Proceeding of a symposium, Braunschweig 1976, 158-170.

Simon-Sylvestre Ginette, 1960. L'enfouissement des pailles dans le sol. Etudes générales et répercussions sur la microflore du sol. Ann. Agron., série A, I, 5-53.

X... 1976. Conséquences agronomiques de l'enfouissement et du brûlage des pailles de céréales. Document I.T.C.F., juillet 1976 , 127-147. 